\title{
LEVELS OF BIOMARKER YKL-40 AND INTERLEUKIN-6 IN GINGIVAL CREVICULAR FLUID IN PATIENTS WITH CHRONIC PERIODONTITIS AND TYPE 2 DIABETES
}

\author{
*Kranti. K1., Pramod. N1., Ashwini. S1 and Shalini Naik² \\ ${ }^{1}$ Department of Periodontology, Faculty of Dental Sciences, Ramaiah University of Applied Science, \\ Bangalore, Karnataka State, India \\ ${ }^{2}$ Faculty of Pharmacy, Ramaiah University of Applied Science, Bangalore, Karnataka state, India \\ DOI: http://dx.doi.org/10.24327/ijrsr.2017.0805.0285
}

\section{ARTICLE INFO}

\section{Article History:}

Received $18^{\text {th }}$ February, 2017

Received in revised form $10^{\text {th }}$

March, 2017

Accepted $06^{\text {th }}$ April, 2017

Published online $28^{\text {th }}$ May, 2017

Key Words:

YKL-40, Interleukin-6, Chronic Periodontitis, Diabetes mellitus, Gingivalcervicular fluid

\begin{abstract}
Elevated YKL - 40 levels are seen in patients with type 2 diabetes.Interleukin- 6 is one of the most studied inflammatory markers in periodontal disease. Aim was to estimate and compare GCF levels of YKL-40 and Interleukin-6 in healthy, chronic periodontitis and type 2 diabetes mellitus patients with chronic periodontitis.82 patients were enrolled into the study and divided into three experimental groups healthy group, chronic periodontitis group and chronic periodontitis with type 2 diabetes mellitus group. Gingivalcrevicular fluid samples were collected and YKL-40 and Interleukin- 6 were analysed by enzyme linked immunosorbent assay. The total amount of YKL-40 and IL-6 in GCF were higher in patients with periodontal tissue breakdown. Within the limits of this study it can be concluded that YKL-40 in GCF may reflect the inflammation of periodontitis and can be a useful marker for inflammation in periodontal diseases and type 2 diabetes mellitus.
\end{abstract}

Copyright (C) Kranti. K et al, 2017, this is an open-access article distributed under the terms of the Creative Commons Attribution License, which permits unrestricted use, distribution and reproduction in any medium, provided the original work is properly cited.

\section{INTRODUCTION}

Acute-phase response occurs in the innate host response to injuries, infections, or ischemic necrosis by releasing various acute-phase proteins. The levels of various acute-phase proteins increase in periodontal disease in both gingival crevicular fluid (GCF) and plasma or serum. Therefore, acute-phase proteins can be susceptibility markers in relation to inflammatory status. ${ }^{1}$

YKL-40, an acute-phase protein, is a novel potential inflammatory marker in relation to both acute and chronic inflammation. ${ }^{1} \quad$ YKL-40 (also known as chitinase-3-like-1 (CHI3L1), human cartilage glycoprotein-39 (HC-gp39) and Chondrex is a $40 \mathrm{kDa}$ heparin, chitin and collagen-binding phylogenetically highly conserved glycoprotein belonging to the family 18 of glycosyl hydrolases comprising chitinases from various species . ${ }^{2,3}$ In vivo YKL-40 protein expression is found in a subpopulation of macrophages in different tissues with inflammation and extracellular matrix remodelling such as macrophages in atherosclerotic plaques, in inflamed synovial membranes of patients with rheumatoid arthritis. YKL-40 increases in patients by more than $25 \%$ following an inflammatory stimulus. Serum or plasma concentrations of YKL-40 are often elevated, compared to healthy subjects, in patients with diseases characterized by inflammation. ${ }^{3}$ Elevated YKL - 40 levels are seen in patients with type 2 diabetes and also increase with increasing levels of albuminaria in type I diabetes. ${ }^{4}$ Significantly increase in the GCF YKL-40 levels and serum YKL-40 levels with severity of periodontal disease is observed. ${ }^{1}$

Interleukin-6 (IL-6) is a multifunctional cytokine synthesized in response to stimuli such as infection and trauma by a variety of cells such as macrophages, neutrophils, keratinocytes, fibroblasts, and endothelial cells. It has been suggested that the main source of salivary IL-6 is the GCF, where it is found at higher concentrations than in salivary glands secretions. ${ }^{5}$

The prevalence of advanced periodontal disease was substantially higher among type II diabetic persons than in non diabetic population. ${ }^{6}$ It is clear that periodontitis is a clear complication of diabetes mellitus. In addition to the recognized increased occurrence and severity of periodontal disease in patients with diabetes, studies have also examined how periodontal therapy affects glycemic control in diabetic patients. 


\section{Kranti. K \&al., Leves of BiomarkerYk-40AndI ntelekin-6I n Gingival Creviaular FluidI nPatientsWith CroricPeriodontitisAndType2Diabetes}

There are limited studies in the literature which evaluated the levels of biomarker YKL-40 in the GCF of diabetic patients. So the aim of the study is to compare the GCF levels of YKL-40 and IL-6 in healthy, chronic periodontitis and chronic periodontitis patients with type 2 diabetes mellitus.

\section{MATERIALS AND METHODS}

A total of 82 subjects between the age group of 25 to 65 years were included in the study. Patients with chronic periodontitis and type 2 diabetes mellitus, having teeth $\geq 20$ were taken for the study. Patient with a history of any systemic disease except type 2 diabetes, smokers, pregnant or lactating mothers, taking antibiotic or other drugs that affect periodontal status in the past 6 months and patients with a history of periodontal treatment in previous 6 months are excluded from the study. Written informed consent from the individuals participating in the study along with ethical clearance from the Institution's Ethical Committee was obtained. Study designis a crosssectional study design. The subjects were divided into 3 groups as follows: Group A: Subjects with healthy periodontium. Group B: Subjects with chronic periodontitis. Group C: Subjects with Type 2 diabetes and chronic periodontitis. All participants underwent a full-mouth periodontal clinical and radiographic examination if they had $\geq 20$ teeth and were classified into groups based on the plaque index (PI) gingival index (GI), probing depth (PD), clinical attachment level (CAL). GCF samples were collected from a site with the highest clinical signs of inflammation and bone loss.

\section{Method of collection of GCF: (Fig: 1)}

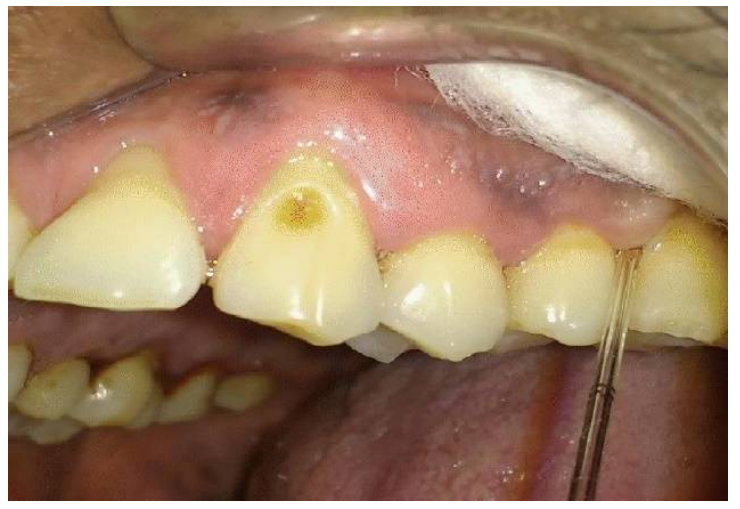

Figure 1 Collection of GCF

GCF samples were collected from a site with the highest clinical signs of inflammation and bone loss. The subjects were asked to rinse their mouth vigorously with water to cleanse the teeth of loosely adherent debris. The sites were carefully dried with a gentle stream of compressed air and isolated. Supragingival plaque was removed to avoid contamination and blocking of the microcapillary pipette. GCF was collected by placing a 1-5 $\mu \mathrm{l}$ calibrated volumetric microcapillary pipette extracrevicularly for 5-20 minutes without touching the marginal gingiva. From each test site a standardized volume of 2-3 $\mu$ l was collected using the calibration on the micropipette. Samples were obtained from the tooth that showed highest amount of clinical attachment loss. The collected GCF is immediately transferred to vials containing $100 \mu \mathrm{l}$ phosphate buffer saline and the samples are stored at $-70^{\circ} \mathrm{C}$ till the time of the assay.
The colorimetric assay procedure was done to measure the YKL-40 and IL-6 levels using a commercially available ELISA kits.(Fig2)

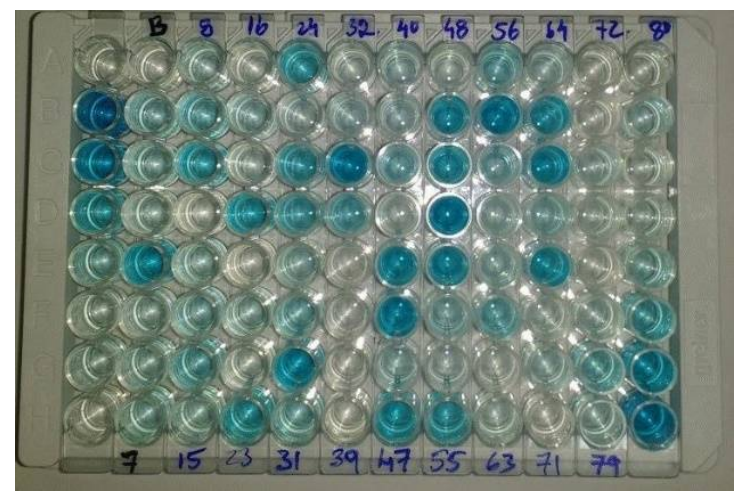

Figure 2 Colorimetric Assay

Statistical analysis: Mean and standard deviation of the continuous variables were calculated. One way Analysis of Variance (Anova) was used to test the difference between groups. The Scheffe post-hock test used relationships between subgroups in pairs of two. The difference in mean was considered statistically significant if $\mathrm{P}<0.05$. Pearson's correlation test was used to evaluate correlation among YKL40 and IL- 6 concentration and the clinical parameters. $\mathrm{P}<0.05$ was considered statistically significant. Data was analysed using SPSS Version 20 package.

\section{RESULTS}

Mean Plaque index scores for group A,B,C were found to be $0.25,2.00,2.03$. (Table 1 ) The difference was found to be statistically significant between group A and B group A and $\mathrm{C}$ but the difference was statistically non significant between group $\mathrm{B}$ and $\mathrm{C}$.

Table 1 Comparison of subject mean values of variables, il-6 and ykl-40 using anova test

\begin{tabular}{ccccccc}
\hline Variable & Group & N & Mean & $\begin{array}{c}\text { Standard } \\
\text { deviation }\end{array}$ & F value & Sig. \\
\hline Plaque & $\mathrm{A}$ & 12 & .25 & .452 & & \\
index & $\mathrm{B}$ & 35 & 2.00 & .000 & 241.327 & .01 \\
& $\mathrm{C}$ & 35 & 2.03 & .296 & & \\
Gingival & $\mathrm{A}$ & 12 & .33 & .492 & & \\
index & $\mathrm{B}$ & 35 & 2.00 & .000 & 421.494 & .01 \\
& $\mathrm{C}$ & 35 & 2.00 & .000 & & \\
Probing & $\mathrm{A}$ & 12 & 1.33 & 1.155 & & \\
depth & $\mathrm{B}$ & 35 & 8.74 & 1.633 & 117.960 & .01 \\
\hline
\end{tabular}

Gingival index scores for group A,B, C were found to be 0.33 , $2.00,200$. (Table 1) The difference was found to be statistically significant between group $\mathrm{A}$ and $\mathrm{B}$ group $\mathrm{A}$ and $\mathrm{C}$ but the difference was statistically non significant between group $\mathrm{B}$ and $\mathrm{C}$.

Probing depth of group A,B,C were found to be 1.33, 8.74 and 8.69. (Table 1) The difference was found to be statistically significant between group A and B and group A and C but the difference was statistically non significant between group $B$ and C. PD showed statistically significant positive correlation with YKL-40. 
Mean Clinical attachment level of groups A,B,C were found to be $1.33,8.69$ and 8 . (Table 1) The difference was found to be statistically significant between group $\mathrm{A}$ and $\mathrm{B}$ and group $\mathrm{A}$ and $\mathrm{C}$ but the difference was statistically non significant between group $\mathrm{B}$ and $\mathrm{C}$.

Mean YKL-40 level of group A,B,C 28.08, 133.34, 222.89. and standard deviation was 144.911.F value was 11.870 . (Table 1) (Graph 1) The difference in mean YKL-40 between all the groups was found to be statistically significant $(\mathrm{P}<0.05)$.

MeanIL-6 levels of group A,B,C were found to be $.83,14.86$ and 11.31. and standard deviation was 5.728.F value was 11.870. (Table 1)(Graph 1) The difference in mean IL-6 between all the groups was found to be statistically significant $(\mathrm{P}<0.05)$. IL-6 was found to be positively correlated with YKL40 in group $\mathrm{A}(\mathrm{r}=.099)$ and negatively correlated to YKL-40 in group B $(r=-.053)$ and $C(r=-.194)$, however the findings were not statistically significant. (Table 2)

Table 2 Pearson correlation coefficient test comparing IL6 with YKL-40 in all groups

\begin{tabular}{clc}
\hline & IL-6 & YKL-40 \\
\hline \multirow{2}{*}{ Group A } & Pearson Correlation & .099 \\
& Sig. & .759 \\
\multirow{2}{*}{ Group B } & Pearson Correlation & -.053 \\
& Sig. & .761 \\
\multirow{2}{*}{ Group C } & Pearson Correlation & -.194 \\
& Sig. & .264 \\
\hline
\end{tabular}
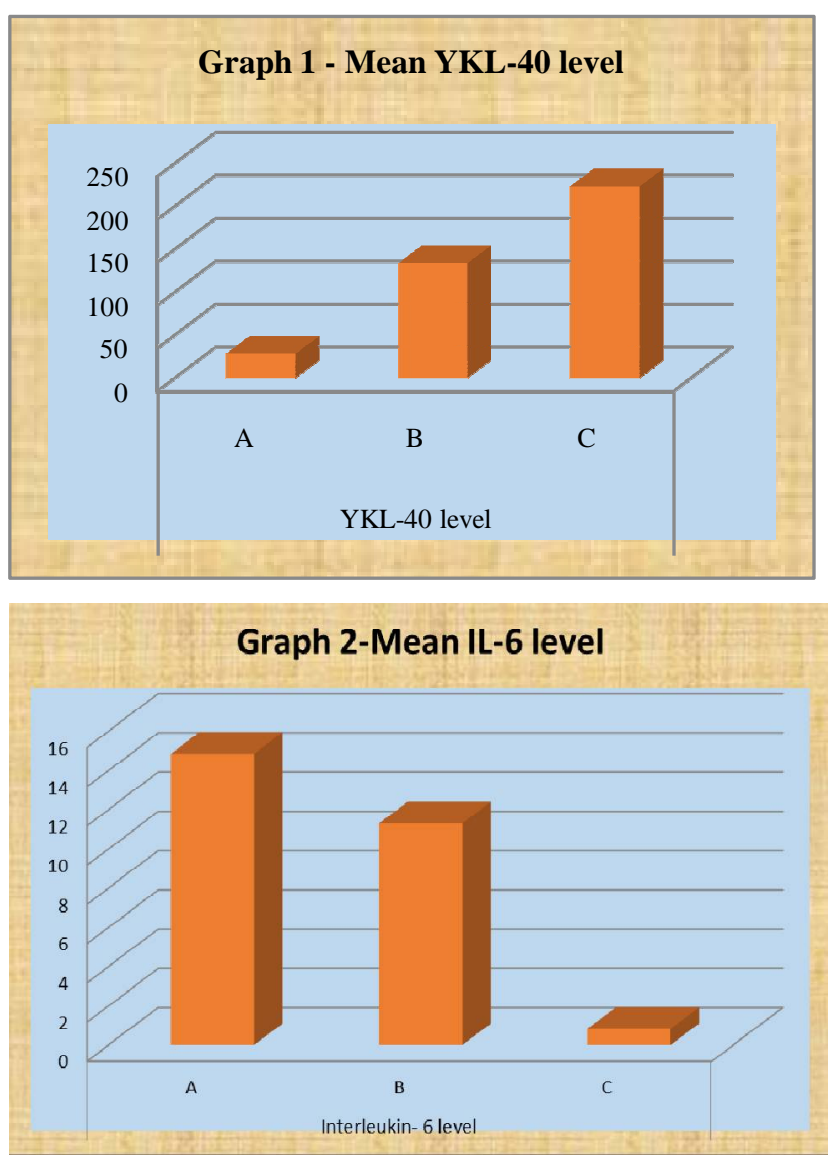

\section{DISCUSSION}

The results of the present study showed that the levels of YKL40 increased progressively from healthy (28.08picogram) to periodontitis patients (133.34picogram) and to diabetes patients with periodontitis (222.89picogram). YKL-40 levels of all the three groups were compared with each other in pairs of two, the difference was found to be statistically significant. There is an increasing clinical evidence which shows that the increased serum or plasma YKL-40 levels in several diseases characterized by inflammation. ${ }^{7-16}$ Data suggest that acutephase reactants might be related to the inflammatory status of periodontal tissues and that YKL-40 is released by a variety of cells, mainly neutrophils and macrophages, that are significantly excessive during periodontal inflammation. ${ }^{7-19}$

In contrast, the present study showed that the mean amount of YKL-40 was less than that of the study of Keles et al (2014) and J. Kido et al (2015). ${ }^{1,20}$ Although the reasons of discrepancies of GCF YKL-40 level and GCF volume were not well known, the differences in a used ELISA kit and method for GCF collection might affect those discrepancies. In the above studies GCF was collected with a filter paper where as in this study micropipettes are used for GCF collection.

In the present study, the mean amount of YKL-40 in GCF samples from the $\mathrm{CP}$ sites was approximately 4.5-fold higher than that of healthy sites, and YKL-40 levels in CP with T2DM showed nearly 2 fold higher levels than the CP group. Our study supports the results of Keles et al (2014) which showed that YKL-40 level was elevated in GCF from sites with periodontitis. Though there is no statistically significant difference in the levels of YKL-40 in periodontitis and diabetic group in study by Kido et al there is overexpression of YKL-40 in GCF of $\mathrm{CP}$ and $\mathrm{CP}$ with T2DM patients than healthy patients.

The present study shows that the total amount of GCF IL-6 levels were higher in patients with $\mathrm{CP}$ and $\mathrm{CP}$ with $\mathrm{T} 2 \mathrm{DM}$ compared with healthy controls. The mean values of IL-6 levels for group A, B, C were 0.83 picogram, 14.86picogram and 11.31 picogram respectively. Studies by Bulent kurtis et al, Becerik. S et al and Takahashi. K et al demonstrated the elevated GCF IL-6 levels in patients with gingivitis and CP. ${ }^{18,19,20}$ Moreover, gingivitis and $\mathrm{CP}$ caused significant increases in the systemic release of acute phase proteins and cytokines. P. M. Duarte at al assessed the levels of IL-6 in gingival tissues of T2DM patients concluded that type 2 diabetes was associated with increased expression of interleukin-6 in periodontally inflamed tissues of diabetic patients and such overexpression may be involved in the mechanisms by which type 2 diabetes enhances periodontal destruction. However, the levels of IL-6 in this study are in contrast to the results of a study by Bulent kurtis et al where more elevated IL-6 levels seen in CP with T2DM than CP. Elevated GCF levels of IL-6 in patients with CP and CP with T2DM in this study support YKL-40 findings, including that patients were in an inflammatory state, and further confirm the role of this cytokine in the pathogenesis of periodontal disease. The composition of GCF is the result of interplay between the bacterial biofilm and cells of the periodontal tissues. In the present study GCF was collected by inserting the pipette at the entrance of the gingival crevice which has an advantage of being non invasive as compared to gingival biopsies. Microcapillary pipettes were used for collection of GCF samples in this study to avoid non specific attachment of the analyte to filter paper fibers, ensuing in false reduction in the 
detectable YKL-40 and IL-6 levels that in turn can underestimate the correlation of levels to disease severity. ${ }^{21}$

The quantitative detection of YKL-40 and IL-6 is performed by ELISA with high sensitivity and specificity. From a clinician's perspective, it is notable that ELISA is generally used for the measurement of human YKL-40 in body fluids (serum, plasma, synovial fluid, and cerebrospinal fluid) and IL-6 in GCF and serum. $^{22,23}$ Data are presented as the total amount of a constituent in GCF instead of its concentration, which is a more appropriate method when examining the relationship between GCF constituents and periodontal diseases. ${ }^{24}$ In the present study, the total amount of YKL-40 and IL-6 levels in GCF is the preferred method of data expression.

In the light of above it is plausible to suggest that total amounts of YKL-40 and IL-6 in GCF were higher in patients with periodontal tissue breakdown. YKL-40 levels in GCF samples from periodontitis increased to approximately 4.5 fold compared with non-diseased levels, twice in CP with T2DM group than $\mathrm{CP}$ group, suggesting that YKL-40 in GCF may reflect the inflammation of periodontitis and can be a useful marker for inflammation in periodontal diseases and type 2 diabetes mellitus.

The variability of YKL-40 and IL-6 concentrations within the patients of each group can be attributed to the severity of inflammation that was present in different stages of disease process at the time of collection of GCF samples, thus implying YKL-40 and IL-6 as a potential biomarker of inflammation in both periodontal disease and diabetes mellitus.

Further, longitudinal prospective studies involving larger population are needed to confirm the findings of the present study and to better understand the role of YKL-40 and IL-6 as a marker of severity of inflammation in pathogenesis of periodontal disease and type 2 diabetes mellitus.

\section{CONCLUSION}

The findings of the study suggest that the total amounts of YKL-40 and IL-6 in GCF were higher in patients with periodontal tissue breakdown. YKL-40 levels in GCF samples from periodontitis increased to approximately 4.5 fold compared to non-diseased levels, twice in CP with T2DM group than CP group, suggesting that YKL-40 in GCF may reflect the inflammation of periodontitis and can be a useful marker for inflammation in periodontal diseases and type 2 diabetes mellitus.

\section{References}

1. Keles ZP, Keles GC, Avci B, Cetinkaya BO, Emingil G.(2014) Analysis of YKL-40 acute-phase protein and interleukin-6 levels in periodontal disease. Journal of periodontology. Sep; 85(9):1240-6.

2. Rathcke CN, Vestergaard H. YKL-40, (2006) A new inflammatory marker with relation to insulin resistance and with a role in endothelial dysfunction and atherosclerosis. Inflammation Research. Jun 1; 55 (6): 221-7.

3. Roslind A, Johansen JS. YKL-40: (2009) A novel marker shared by chronic inflammation and oncogenic transformation. Inflammation and Cancer: Methods and
Protocols: Volume 1: Experimental Models and Practical Approaches. 159-84.

4. Persson F, Rathcke CN, Gall MA, Parving $\mathrm{HH}$, Vestergaard H, Rossing P.(2012) High YKL-40 levels predict mortality in patients with type 2 diabetes. Diabetes research and clinical practice. Apr 30; 96(1):84-9.

5. Nibali L, Fedele S, D'aiuto F, Donos N. Interleukin-6 in oral diseases: (2012) A review. Oral diseases. Apr 1; 18(3):236-43.

6. Löe H. Periodontal disease: the sixth complication of diabetes mellitus. Diabetes care. 1993 Jan 1; 16(1):32934.

7. Johansen JS, Jensen HS, Price PA. (1993) A new biochemical marker for joint injury. Analysis of YKL-40 in serum and synovial fluid. Rheumatology. Nov 1; 32(11):949-55.

8. Létuvé S, Kozhich A, Arouche N, Grandsaigne M, Reed J, Dombret MC, Kiener PA, Aubier M, Coyle AJ, Pretolani M.(2008) YKL-40 is elevated in patients with chronic obstructive pulmonary disease and activates alveolar macrophages. The Journal of Immunology. Oct 1; 181(7):5167-73.

9. Craig-Schapiro R, Perrin RJ, Roe CM, Xiong C, Carter D, Cairns NJ, Mintun MA, Peskind ER, Li G, Galasko DR, Clark CM.(2010 ) YKL-40: a novel prognostic fluid biomarker for preclinical Alzheimer's disease. Biological psychiatry. Nov 15; 68(10):903-12.

10. Vind I, Johansen JS, Price PA, Munkholm P.(2009) Serum YKL-40, a potential new marker of disease activity in patients with inflammatory bowel disease. Scandinavian journal of gastroenterology. Jul 8.

11. Kucur M, Isman FK, Karadag B, Vural VA, Tavsanoglu S.(2007) Serum YKL-40 levels in patients with coronary artery disease. Coronary artery disease. 2007 Aug 1; 18(5):391-6.

12. Wang Y, Ripa RS, Johansen JS, Gabrielsen A, Steinbrüchel DA, Friis T, Bindslev L, Haack-Sørensen M, Jørgensen E, Kastrup J.(2008) YKL-40 a new biomarker in patients with acute coronary syndrome or stable coronary artery disease. Scandinavian Cardiovascular Journal. 2008 Jan 1; 42(5):295-302.

13. Lin $\mathrm{CH}$, Li HY, Jiang YD, Chang TJ, Chuang LM.(2013) Plasma YKL-40 predicts 10-year cardiovascular and all-cause mortality in individuals with type 2 diabetes. Clinical endocrinology. 2013 Aug 1;79(2):185-91.

14. Nielsen AR, Erikstrup C, Johansen JS, Fischer CP, Plomgaard P, Krogh-Madsen R, Taudorf S, Lindegaard B, Pedersen BK. Plasma(2008) YKL-40 A BMIindependent marker of type 2 diabetes. Diabetes. 2008 Nov 1; 57(11):3078-82.

15. Rathcke CN, Johansen JS, Vestergaard H. YKL-40, (2006) a biomarker of inflammation, is elevated in patients with type 2 diabetes and is related to insulin resistance. Inflammation Research. 2006 Feb 1; 55(2):53-9.

16. Rathcke CN, Persson F, Tarnow L, Rossing P, Vestergaard H. YKL-40, a marker of inflammation and endothelial dysfunction, is elevated in patients with type 
1 diabetes and increases with levels of albuminuria. Diabetes Care. 2009 Feb 1; 32(2):323-8.

17. PolePalle T, SrinivaSMoogala SB, PeSala DS, Palagi FB.(2015) Acute phase proteins and their role in periodontitis: a review. Journal of clinical and diagnostic research: JCDR. 2015 Nov; 9(11):ZE01.

18. Takahashi K, Takashiba S, Nagai A, (1994) Assessment of interleukin-6 in the pathogenesis of periodontal disease. J Periodontol 1994; 65:147-153.

19. Becerik S, Ozcxaka O, Nalbantsoy A, et al (2010). Effects of menstrual cycle on periodontal health and gingival crevicular fluid markers. J Periodontol 2010; 81:673681.

20. Kido J, Bando Y, Bando M, Kajiura Y, Hiroshima Y, Inagaki $Y$, Murata $H$, Ikuta $T$, Kido R, Naruishi $K$, Funaki M.(2015 ) YKL-40 level in gingival crevicular fluid from patients with periodontitis and type 2 diabetes. Oral diseases. 2015 Jul 1; 21(5):667-73.
21. Okada H, Murakami S (1998). Cytokine expression in periodontal health and disease. Crit Rev Oral Biol Med. 1998; 9(3):248-66.

22. Shimada Y, Komatsu Y, Ikezawa-Suzuki I, Tai H, Sugita N, Yoshie H.(2010) The effect of periodontal treatment on serum leptin, interleukin-6, and C-reactive protein. J Periodontol 2010; 81:1118-1123.

23. Lamster IB, Oshrain RL, Fiorello LA, Celenti RS, Gordon JM.(1988) A comparison of 4 methods of data presentation for lysosomal enzyme activity in gingival crevicular fluid. J ClinPeriodontol 1988; 15:347-352.

24. Hirano T, Matsuda T, Turner M, Miyasaka N, Buchan G, Tang B, Sato K, Shimi M, Maid R, Feldmann M, Kishimoto T.(1998) Excessive production of interleukin 6/B cell stimulatory factor-2 in rheumatoid arthritis. European journal of immunology. 1988 Nov 1; 18(11):1797-802.

\section{How to cite this article:}

Kranti. K et al.2017, Levels of Biomarker Ykl-40 and Interleukin-6 In Gingival Crevicular Fluid In Patients With Chronic Periodontitis and Type 2 Diabetes. Int J Recent Sci Res. 8(5), pp. 17115-17119.

DOI: http://dx.doi.org/10.24327/ijrsr.2017.0805.0285 